Aims and objectives Compare 3 month survival of patients with metastatic cancer admitted to hospice and hospital.

Methods All adults known to have metastatic cancer preadmission to district general hospital or hospice over a 2 month period were included. Parameters compared: proportions discharged and died; length of in-patient stay; mean survival; survival at 3 months.

Results Total number of patients - 106 (hospital:72; hospice:34). $60 \%$ of hospital group women; $65 \%$ of hospice men. Hospice patients were significantly younger, mean age 69.09 vs 75.36 years; p-value 0.006. There was no difference in baseline investigation (FBC; renal and liver functions; serum calcium) results of two groups. There was no difference in proportion of patients discharged $(57 \%$ hospital vs $59 \%$ hospice); proportion dying during admission (43\% hospital vs $41 \%$ hospice); mean or median length of in-patient stay (hospital vs hospice, mean days: 13.29 vs 14.26 , median -9.5 vs 9.5 ); and mean survival (22 days) in 2 groups. Forty four percent of hospital patients were alive at 3 months, versus $35 \%$ of hospice - but insignificant difference (p-value: 0.121). There was no correlation between age and survival $(r=-0.10$; p-value: 0.941).

Conclusions This small study demonstrates no survival benefit of invasive (district general) hospital approach against holistic hospice approach in patients with metastatic cancer. Hospital clinicians should not be concerned that hospice approach would shorten survival of their patients and lengthen hospital stay. All clinicians and managers should be aware that a third of hospice patients survive more than 3 months and that there are mechanisms to identify and manage them promptly.

\section{P-81 GLASGOW PROGNOSTIC SCALE: USEFULNESS IN PREDICTING 3-MONTH SURVIVAL IN METASTATIC CANCER PATIENTS}

\footnotetext{
${ }^{1,2}$ Sanjay Shah. ${ }^{1}$ Northamptonshire Healthcare NHS Foundation Trust, Kettering, UK; ${ }^{2}$ Kettering General Hospital NHS Foundation Trust
}

\subsection{6/bmjspcare-2017-00133.80}

Background In England, many end of life care or hospice-athome services will provide care for patients with life expectancy of $<8-12$ weeks. Low serum albumin has been associated with poor survival in carcinoma of stomach. Raised serum C-reactive protein (CRP) levels have also been linked to poor survival in cancer patients. Glasgow Prognostic Score (GPS) combines serum albumin and CRP levels to give a composite score. Poor survival is associated with high GPS in colorectal cancer patients.

Aims and objectives To compare 3 month survival of patients with metastatic cancer using GPS

Methods All adults known to have metastatic cancer preadmission to district general hospital and hospice over a 2 month period were included. Using serum albumin $(>35 \mathrm{gm} /$ $\mathrm{L}=0 ;<=35 \mathrm{gm} / \mathrm{L}=1)$ and serum CRP $(<5 \mathrm{mg} / \mathrm{L}=0 ;>5 \mathrm{mg} /$ $\mathrm{L}=1$ ), GPS score was assigned (minimum 0 ; maximum 2). Survival duration, to a maximum follow-up of 3 months, was measured. Survival proportions were compared for GPS scores of 0,1 and 2 .

Results Total number of patients - 106. Women comprised $52 \%$. Mean age was 73.6 years. Common cancers: lung $(n=26)$, urology $(n=14)$, breast $(n=12)$ and colorectal, upper gastrointestinal and hepatobiliary-pancreas ( 9 each). GPS could be calculated for 79: 0 in one patient, 1 in 33 and 2 in 45 . Whereas $36 \%$ patients with GPS of 1 died during the admission, $47 \%$ of those with GPS of 2 died. At 3 months respective figures were $42 \%$ and $62 \%$. Mean survival was 17.69 days with GPS 2; and 28.20 days with GPS 1 . The sole patient with GPS 0 died during the inpatient stay.

Conclusions Higher GPS is associated with shorter survival in unselected metastatic cancer patients. This study is too small to determine sensitivity and specificity. A larger study for this inexpensive, widely available and easy to use tool is required.

\section{P-82 WHAT LEVEL OF MEDICAL INPUT DO HOSPICE INPATIENTS NEED AND DOES THIS CORRESPOND TO THEIR NEED FOR SPECIALIST NURSING INPUT? A SERVICE EVALUATION IN A UK HOSPICE}

${ }^{1,2}$ Craig Gannon. ${ }^{1}$ Princess Alice Hospice, Esher, UK; ${ }^{2}$ University of Surrey, Guildford, UK

\subsection{6/bmjspcare-2017-00133.81}

Introduction Though medical input to hospice inpatients is well-established, the evidence detailing the nature and level of medical staffing is lacking. Moreover, there are calls for hospices to develop less medical, more public health models.

To facilitate nursing skill-mix changes, and broaden our 'reach' (to include 'low-complexity' patients), our inpatient unit was split equally into generalist nursing (GN) and specialist nursing ( $\mathrm{SN}$ ) beds. It was suggested $\mathrm{GN}$-suitable patients would correspondingly have few medical needs. To facilitate medical workforce planning, we wanted to evaluate the medical needs across these potentially contrasting populations.

Methods We developed a tool to detail the nature and intensity of medical interventions. We completed a 1 month prospective pilot, in a 28-bedded UK hospice, scoring perceived patient need each day.

Results A tool reflecting overall medical need was generated; with 3-ratings (low, moderate, high), across 9 items (e.g. urgency, clinical complexity, trajectory, discord).

284 patient assessments were completed (100\%); the range of medical need for patients in GN beds was; low $=78$, moderate $=41$, high $=14$ and for $\mathrm{SN}$ beds; low $=41$, moderate $=63$, high $=46$. Concordance of medical and nursing complexity for GN patients $=58 \%$ and SN patients $=31 \%$

Discussion A spectrum of need for medical input to hospice inpatients was confirmed; the level fluctuated during a patient's stay and high needs were not restricted to $\mathrm{SN}$ patients.

The limited concordance between a patient's perceived need for medical input and their suitability for SN or GN, questioned the wider applicability of this differentiation. There was a trend for lower medical input for ' $\mathrm{GN}$ ' compared to 'SN patients'. However, 41\% of 'GN patients' had moderate or high medical needs and only $31 \%$ of 'SN patients' had high medical needs.

The pilot tool appeared suitable for benchmarking the need for medical input; informing our workforce planning and warranting further evaluation, to include other care settings. 